\title{
Ensemble Methods for Tracking and Segmentation (Abstract)
}

\author{
Shai Avidan \\ School of Electrical Engineering, \\ Tel-Aviv University, \\ Tel-Aviv 69978, Israel \\ avidan@eng.tau.ac.il \\ http://www.www.tau.ac.il/avidan
}

\begin{abstract}
Ensemble methods offer an elegant way of training an ensemble of weak classifiers into a strong classifier through the use of the AdaBoost algorithm. In this abstract we discuss two extensions of AdaBoost and demonstrate them on two problems in the field of Computer Vision. The first, termed Ensemble Tracking, extends AdaBoost in the temporal domain and adapts it to the problem of tracking an object in a video sequence. The second, termed SpatialBoost, extends AdaBoost in the spatial domain and adapts it to the problem of interactive image segmentation.

In the case of Ensemble Tracking, we consider tracking as a binary classification problem, where an ensemble of weak classifiers is trained online to distinguish between the object and the background. But because of real-time constraints we can only train one weak classifier per frame and can not maintain an ensemble of more than a limited number of weak classifiers. We propose an online extension of AdaBoost, the Ensemble Tracker, that constantly, and efficiently, combines this stream of weak classifiers into a strong classifier, without going beyond the bound on the number of weak classifiers.

In the case of SpatialBoost we show how to extend AdaBoost to incorporate spatial reasoning and demonstrate it on the problem of interactive image segmentation. The user marks some of the pixels as positive and negative examples and then lets the algorithm label the rest of the pixels. Simply training AdaBoost on the appearance of the labeled pixels and using it to label the unlabeled pixels yields unsatisfactory results. This is because AdaBoost lacks spatial reasoning. But in fact, we know that nearby pixels should, quite often, have the same label. To this end we introduce spatial reasoning in the form of weak classifiers that attempt to infer pixel label from the pixel labels of surrounding pixels, after each boosting iteration. SpatialBoost combines these spatial weak classifiers with the appearance based weak classifier to give superior results.

Taken together, these extensions demonstrate the flexibility of ensemble methods, and the ways in which they can be modified to account for special properties of images and video. We conclude by sketching a number of possible extensions to this line of work.
\end{abstract}

